\title{
IS THE BRAIN NATRIURETIC PEPTIDE A \\ PREDICTOR OF NEW ATRIAL FIBRILLATION IN \\ THE POSTOPERATIVE CARE OF PATIENTS UNDERGOING LUNG RESECTION?
}
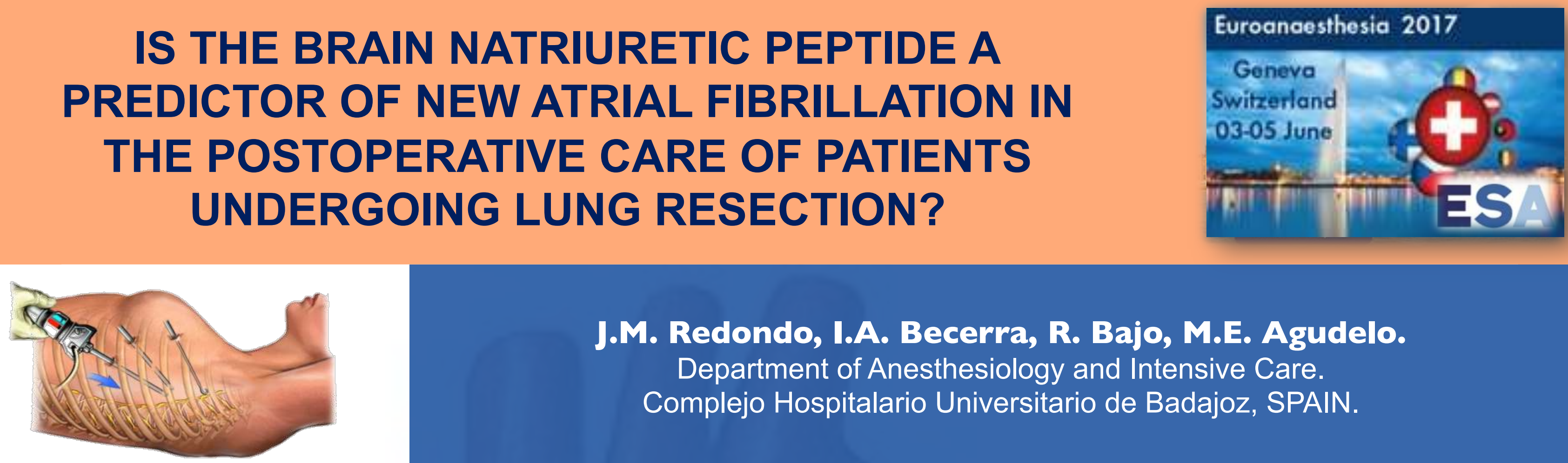

\author{
J.M. Redondo, I.A. Becerra, R. Bajo, M.E. Agudelo. \\ Department of Anesthesiology and Intensive Care. \\ Complejo Hospitalario Universitario de Badajoz, SPAIN.
}

\section{Background and goal of study}

Atrial fibrillation is one of the more common complications of thoracic and esophageal surgery. It occurs in between $12-39 \%$ of patients who undergo a lung resection. . This study aimed to investigate a possible relationship between preoperative Brain Natriuretic Peptide (pro-BNP) and presence of atrial fibrillation in the early postoperative period after lung resection.

\section{Material and methods}

This was a prospective observational study of patients $(n=62)$ who underwent any kind of lung resection surgery (thoracotomy and VATS) in our hospital from January to December 2016. Exclusion criteria were history of previous arrhythmia and lack of informed consent. The following parameters were recorded: demographic characteristics, kind of lung resection surgery, epidural or intravenous postoperative analgesia, preoperative study (ASA physical status classification system, cardiovascular risk factors, pulmonary function tests, baseline arterial blood gas, electrocardiogram, Brain Natriuretic Peptide and haemoglobin), 30-day ocurrence of postoperative atrial fibrillation (primary endpoint) and total hospital stay. The study was carried out after the approval of the Hospital Ethic Committe. The data were analyzed with $95 \%$ confidence by SPSS20, using descriptive and analytic statistics.

\section{Results and discussion}

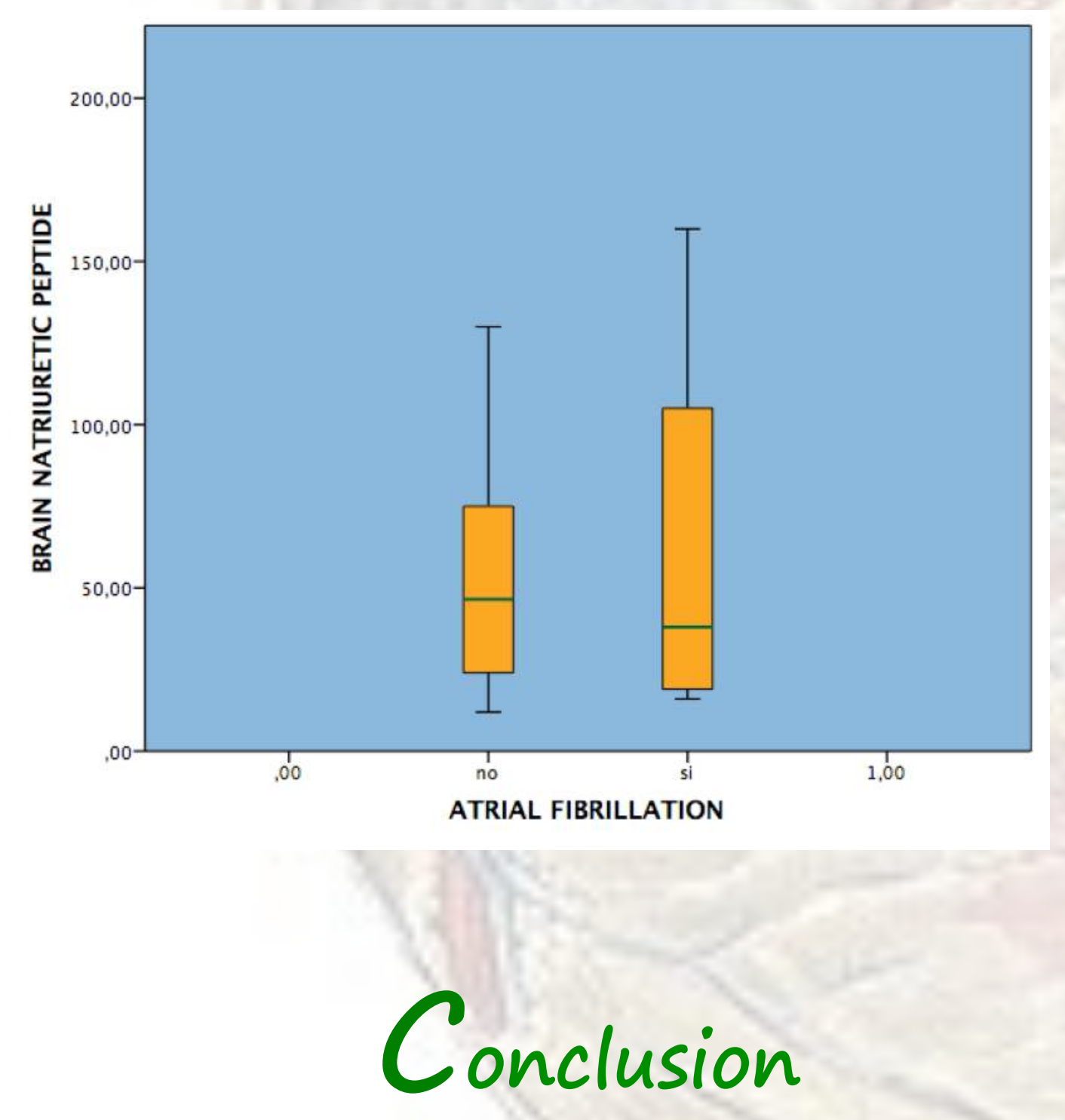

Atrial fibrillation is the most common medical complication after thoracic surgery. BNP was not able to be considered as a good predictor of new atrial fibrillation in our lung resection sample. Optimization and study of cardiovascular risk factors in this type of patients is essential.

\begin{abstract}
A total of 62 lung resections were performed (19\% pneumonectomies, 50\% lobectomies, 10\% segmentectomies and $20 \%$ wedge resections). We registered 5 cases of 30 -day postoperative atrial fibrillation (60\% left neumonectomies, $20 \%$ right upper lobectomy and $20 \%$ left upper lobectomy). All cases were observed in men with cardiovascular risk factors, in the second day after thoracotomy and all of them received epidural analgesia in postoperative period. 30-day postoperative atrial fibrillation rate of incidence was 8 cases per 100 surgery-year in 2016. No statistically significant association between BNP and atrial fibrillation cases was founded $(p=0.91)$. The annual median length of hospital stay was 9 days after lung resection.
\end{abstract}

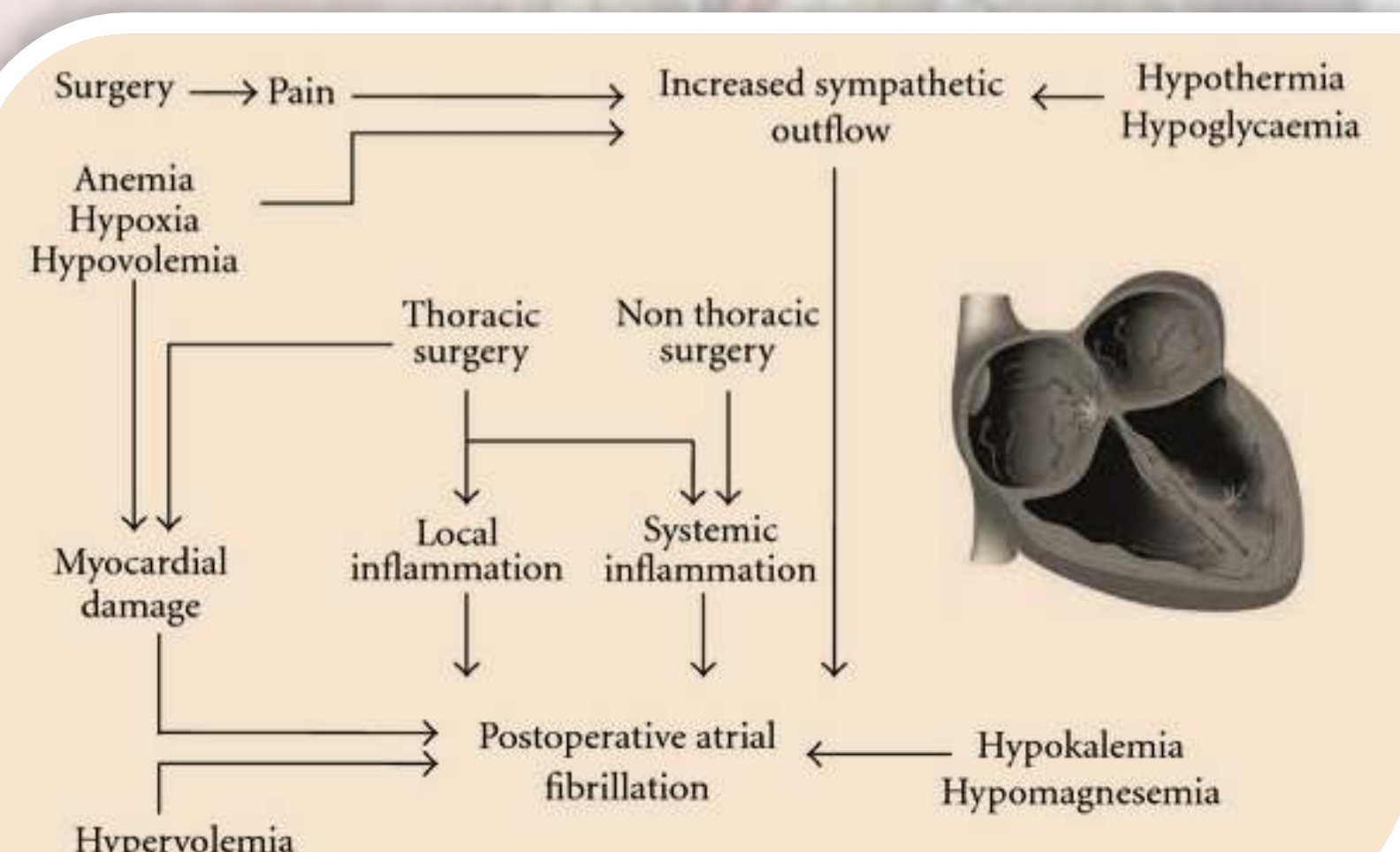

